\title{
Cost-effectiveness analysis of additional docetaxel for metastatic hormone-sensitive prostate cancer treated with androgen-deprivation therapy from a Chinese perspective
}

H.R. ZHENG, BaChelor, pharmacist, Department of Clinical Pharmacy, West China Hospital, Sichuan University, Sichuan, China, F. WEN, Master, PHD Candidate, Department of Medical Oncology, Cancer Center, State Key Laboratory of Biotherapy, West China Hospital, Sichuan University, Sichuan, China, Y.F. WU, MPH, School of Public Health, University of Michigan, Ann Arbor, MI, J.R.C. WHEELER, PHD, Professor emeritus, School of Public Health, University of Michigan, Ann Arbor, MI, USA, \& Q. LI, MD, PHD, PRofessor, Department of Medical Oncology, Cancer Center, State Key Laboratory of Biotherapy, West China Hospital, Sichuan University, Sichuan, China

ZHENG H.R., WEN F., WU Y.F., WHEELER J.R.C. \& LI Q. (2017) European Journal of Cancer Care 26, e12505, doi: 10.1111/ecc.12505

Cost-effectiveness analysis of additional docetaxel for metastatic hormone-sensitive prostate cancer treated with androgen-deprivation therapy from a Chinese perspective

The E3805 (CHAARTED) study found that docetaxel combined with androgen-deprivation therapy (ADT) significantly improved overall survival of patients with metastatic hormone-sensitive prostate cancer. This study aims to determine whether docetaxel combined with ADT is a cost-effective strategy for advanced prostate cancer in China. According to the E3805 study, two groups (docetaxel + ADT and ADT alone) and three health states [progression-free survival (PFS), progressive disease (PD) and death] were analysed in a Markov model. All medical costs were calculated from the Chinese societal perspective. Quality-adjusted life year (QALY) and incremental cost-effectiveness ratios (ICERs) were applied as the primary outcome. Overall, the addition of docetaxel was estimated to increase the cost by $\$ 12816.93$, with a gain of 0.48 QALY. Additionally, for patients with high-volume disease, the increased cost and effectiveness were $\$ 14627.75$ and 0.69 QALYs in docetaxel + ADT group versus the ADT alone group, and the ICER was \$21 199.63 per QALY. These ICERs are far more than the commonly accepted willingness-to-pay (WTP) threshold of \$20 301 per QALY in China. In spite of longer survival time, docetaxel combined with ADT is not a recommended costeffective treatment for metastatic hormone-sensitive prostate cancer in the Chinese setting.

Keywords: cost-effectiveness, prostate cancer, docetaxel, androgen-deprivation therapy.

Correspondence address: Qiu Li, Department of Medical Oncology, Cancer Center, State Key Laboratory of Biotherapy, West China Hospital, Sichuan University, Sichuan, China (e-mail: fbqiu9@163.com). Hanrui Zheng, Feng Wen and Yifan Wu contributed equally to this work.

Accepted 18 March 2016

DOI: $10.1111 /$ ecc. 12505

European Journal of Cancer Care, 2017, 26, e12505, DOI: 10.1111/ ecc. 12505

\section{INTRODUCTION}

Prostate cancer is the second most frequently diagnosed cancer in men, and ranks fifth in estimated deaths worldwide (Siegel et al. 2015). In China, the incidence is increasing, reflecting risk factors including an increased consumption of animal fats, obesity and physical inactivity, which are associated with economic development (Potosky et al. 1995; Center et al. 2012; Siegel et al. 2015). Although death rates for prostate cancer have been 
decreasing in the majority of developed countries (a result of early detection and improved treatment), mortality rates are rising in some Asian and Central and Eastern European countries, including China, with an estimated 5 -year survival rate at 54\% (Potosky et al. 1995; Center et al. 2012; Hongmei et al. 2015; Siegel et al. 2015).

The majority of prostate cancer-related deaths result from metastatic spread of prostate cancer cells from the primary tumour to contiguous and distal sites (Maluf et al. 2012). Targeting the androgen receptor axis has been long known as an effective strategy in metastatic prostate adenocarcinoma, and androgen-deprivation therapy (ADT) has been the mainstay of treatment for metastatic prostate cancer since the 1980s (Jr et al. 1987). Surgical excision of the testes or the use of drugs to interrupt signalling between the pituitary gland and the testes are curative options of ADT (Nishiyama 2008). However, a recent study demonstrated limited improvement in overall survival (OS) for patients diagnosed with metastatic hormone-sensitive prostate cancer treated with ADT (Wu et al. 2014). Subsequently, the TAX 327 study showed that the OS of patients with hormone-resistant prostate cancer treated with docetaxel plus prednisone was statistically longer than those treated with mitoxantrone and prednisone (19.2 versus 16.3 months; $P=0.004$; Tannock et al. 2004). Hence, docetaxel was approved by the US Food and Drug Administration for castration-resistant prostate cancer. However, whether docetaxel can improve outcomes for metastatic hormone-sensitive patients was still unknown.

Most recently, the E3805 (CHAARTED) trial, which compared ADT alone and ADT plus docetaxel for metastatic hormone-sensitive prostate cancer, showed that 6 cycles of docetaxel at the beginning of ADT for metastatic prostate cancer resulted in significantly longer OS than that with ADT alone $(57.6$ months versus 44.0 months; $P<0.001$ ). In the subgroup with high-volume disease (presence of visceral metastases or $\geq 4$ bone lesions with $\geq 1$ beyond the vertebral bodies and pelvis), the benefit was even greater, with a median 17.0 months longer OS in the combination group than in the ADT alone group [hazard ratio $(\mathrm{HR})=0.60 ; P<0.001$ ] (Sweeney et al. 2015).

Despite the obvious benefit in OS, ADT plus docetaxel, compared to $\mathrm{ADT}$, has been found to increase the incidence of various side effects, including grade 3 or 4 neutropenia, anaemia, thrombocytopenia and febrile neutropenia (Sweeney et al. 2015). In addition to adverse drug reactions, the use of docetaxel may cause an additional monetary burden for patients, especially those in developing countries, such as China. Thus, it is critical to identify whether docetaxel is a cost-effective option for patients with metastatic hormone-sensitive prostate cancer. The aim of this study was to conduct a cost-effectiveness analysis using a Markov decision model to compare $\mathrm{ADT}$ and ADT plus docetaxel for metastatic hormonesensitive prostate cancer patients based on the E3805 trial from the Chinese societal perspective.

\section{MATERIALS AND METHODS}

\section{Patients}

The patient data analysed in the current study was originally from the E3805 trial, which found the advantage of $\mathrm{ADT}$ and docetaxel concomitant therapy compared with $\mathrm{ADT}$ alone in the treatment of metastatic, hormone-sensitive prostate cancer. In short, 790 qualified patients with histologically or clinically confirmed prostate cancer, and radiological evidence of metastatic disease were included in the research. High-volume metastasis was defined as the presence of visceral metastases or $\geq 4$ bone lesions with $\geq 1$ beyond the vertebral bodies and pelvis (Sweeney et al. 2015). Among the patients, 397 were in the ADT plus docetaxel group and 393 in the ADT alone group. The median age was 63, and their Eastern Cooperative Oncology Group performance status ranged from 0 to 2 .

\section{The treatments}

The clinical trial has been described elsewhere (Sweeney et al. 2015). In brief, all patients were required to take calcium carbonate at least $500 \mathrm{mg}$ per day and vitamin D no less than 400 IU every day. Every patient received ADT according to the protocol (Sweeney et al. 2015). For ADT plus docetaxel group, docetaxel was given at a dose of $75 \mathrm{mg} / \mathrm{m}^{2}$ of body surface area every 21 days for maximum of 6 cycles; a dose of $8 \mathrm{mg}$ oral dexamethasone was taken 12,3 and $1 \mathrm{~h}$ prior to docetaxel infusion. The response evaluation was conducted, and grade 3 or higher toxic effects were captured (Sweeney et al. 2015). Treatments of the second-line included cabazitaxel, mitoxantrone, abiraterone, antiandrogen (flutamide) and docetaxel, as demonstrated in the E3805 study (Sweeney et al. 2015).

\section{Clinical outcomes}

For all participants in the study cohort, the median OS was 57.6 months in the combination group compared with 44.0 months in the ADT group [HR $=0.61 ; 95 \%$ confidence interval (CI), 0.47-0.80; $P<0.001]$. Notably, the survival benefit was more significant in the high-volume disease patients, which was 49.2 months in the combina- 
tion group and 32.2 months in the ADT alone group $(\mathrm{HR}=0.60 ; 95 \% \mathrm{CI}, 0.45-0.81 ; P<0.001)$. Among the combination therapy patients, the grade 3 or 4 febrile neutropenia rate was $6.2 \%$, the grade 3 or 4 infection with neutropenia rate was $2.3 \%$, and the grade 3 sensory neuropathy and grade 3 motor neuropathy were $0.5 \%$ (Sweeney et al. 2015).

\section{Overall concept of the Markov model}

To evaluate the economic consequences and therapeutic efficacy associated with each testing item and treatment strategy, a Markov decision model was built using Treeage software (Treeage, Williamstown, MA, USA). The time horizon was 10 years, which was nearly lifelong and the transition cycle length was 1 year. The transition diagram among model states is presented in Figure 1. The costs were calculated from the Chinese societal perspective, and survival was reported in quality-adjusted life years (QALYs). The incremental cost-effectiveness ratios
(ICERs) were calculated as the difference in costs divided by the difference in effectiveness between a given strategy and the next most cost-effective alternative.

\section{The strategies and Markov model structure}

Two groups were analysed: patients treated with docetaxel and ADT combination therapy and patients treated with $\mathrm{ADT}$ alone. Three health states were analysed: progressionfree survival (PFS), progressive disease (PD) and death (Fig. 1). A patient was assumed to enter the model at the PFS state and received either docetaxel and $\mathrm{ADT}$ combination therapy or ADT alone as the first-line treatments until one of the following events occurred: progression of disease (PD stage), intolerable toxicities or death. The transition probabilities of health states were estimated based on an equation used pre-

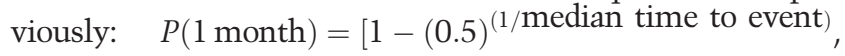
which was derived from the equations below: $P=1-e^{-R}$ and $R=-\ln [0.5] /($ time to event/number of treatment cycles) (Purmonen et al. 2008; Petrou \& Talias 2014).

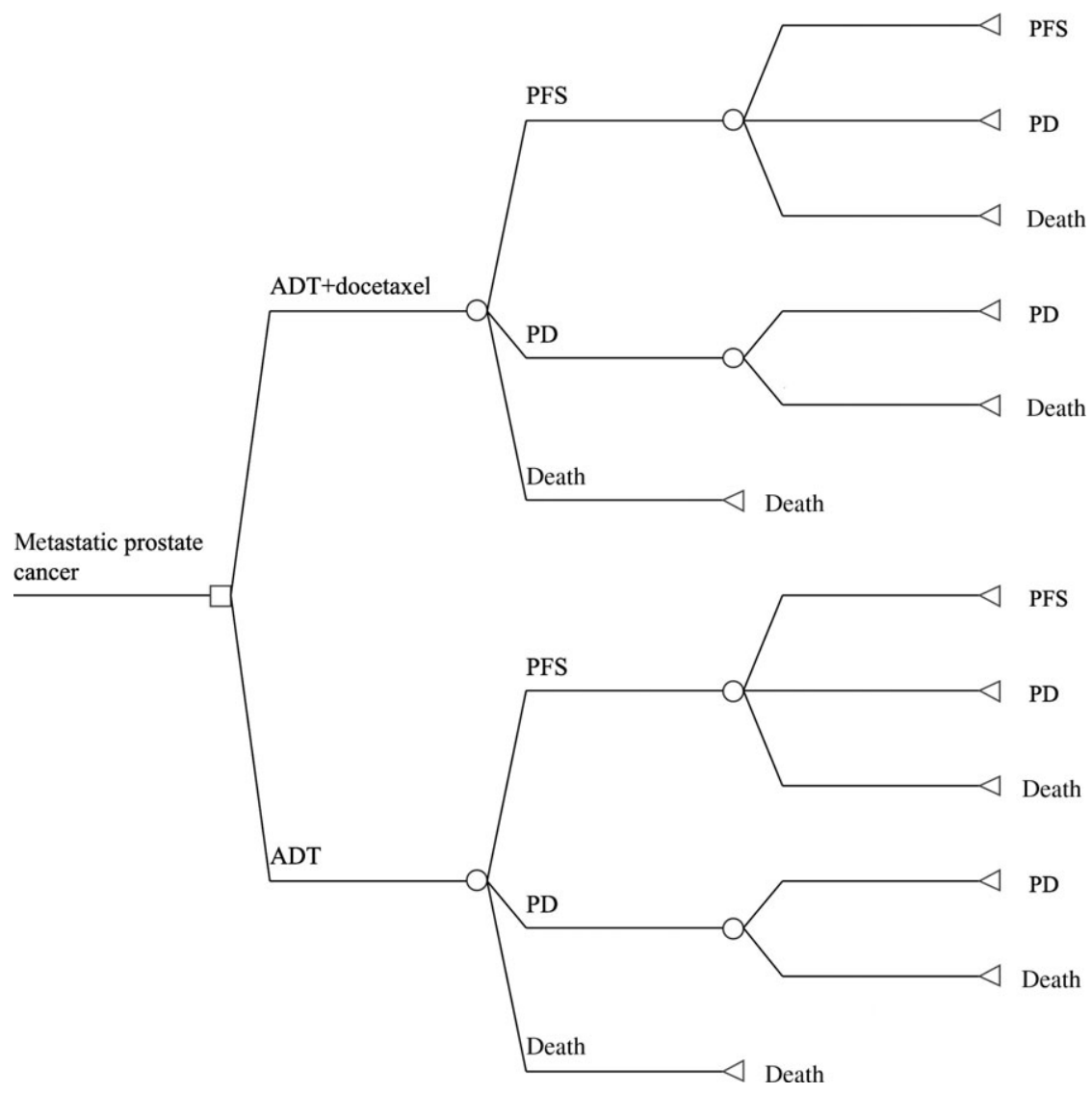

Figure 1. Markov model for metastatic prostate cancer patients based on the trial of E3805. According to the E3805 profile, two groups were analysed: group 1, patients with metastatic prostate cancer treated with ADT + docetaxel; group 2, patients with metastatic prostate cancer treated with ADT. A Markov model comprising three health states (PFS, PD and death) was built. ADT, androgen-deprivation therapy; PD, progression disease; PFS, progression-free survival. 
Table 1. Transition probabilities used in the analysis

\begin{tabular}{llll}
\hline & Value & Lower limit & Higher limit \\
\hline ADT+D group & & & \\
pPFS-PFS-1 & 0.967 & 0.774 & 1.000 \\
pPFS-PD-1 & 0.021 & 0.017 & 0.025 \\
pPFS-death-1 & 0.012 & 0.010 & 0.014 \\
pPD-PD-1 & 0.972 & 0.778 & 1.000 \\
pPD-death-1 & 0.028 & 0.022 & 0.034 \\
ADT group & & & \\
pPFS-PFS-2 & 0.95 & 0.760 & 1.000 \\
pPFS-PD-2 & 0.034 & 0.027 & 0.041 \\
pPFS-death-2 & 0.016 & 0.013 & 0.019 \\
pPD-PD-2 & 0.972 & 0.778 & 1.000 \\
pPD-death-2 & 0.028 & 0.022 & 0.034 \\
\hline
\end{tabular}

PFS, progression-free survival; PD, progressive disease; ADT, androgen-deprivation therapy; $\mathrm{D}$, docetaxel.

Based on the equation, monthly transition probability from PFS state to PFS state ( $p$ PFS-PFS), from the PFS to PD ( $p$ PFS-PD), from PFS to death ( $p$ PFS-death), from PD to PD $(p \mathrm{PD}-\mathrm{PD})$ and from $\mathrm{PD}$ to death ( $p$ PD-death) are described in Table 1.

\section{The utilities}

We calculated the effectiveness data in each group based on the health-related quality of life. Preference-based health states utility scores were derived from previously published studies and the values were set at 0.8 for PFS state, 0.6 for PD state and 0 for death respectively (Heijnsdijk et al. 2015). Since the patients' performance status of the treatments in two groups was very similar, the same utility values were applied in the model.

\section{Measurement of costs}

Costs were calculated from the Chinese societal perspective. Costs for the first-line therapies as well as secondline treatments were included in the analysis. Both direct costs and indirect costs were taken into account. Direct costs were associated with costs of drugs, hospitalisation and necessary tests for efficacy/safety evaluation during the treatments. Indirect costs included costs related to grade 3-4 adverse events (AEs), and calcium carbonate and vitamin D costs. Detailed data on the grade 3-4 AEs were derived from the records of E3805 study. All costs were converted into US dollars, with an exchange rate of $\$ 1=¥ 6.39$ (26 August 2015).

\section{Cost-effectiveness analysis}

Based on the data collected above, a cohort of 100000 patients was simulated randomly, using the Monte Carlo simulation to imitate the process of metastatic prostate cancer. The model was run until all the hypothetical patients died. Costs and effectiveness were discounted at $3 \%$ per year.

\section{Subgroup analyses}

We also conducted subgroup analyses based on the extent of metastases. The efficacy data of patients with high/lowvolume disease were derived from the Kaplan-Meier survival curves of patients in the subgroups. The costeffectiveness data of addition of docetaxel to ADT compared with ADT alone in the subgroups were measured according to the methods mentioned above.

\section{Sensitivity analysis}

One-way sensitivity analysis was performed to examine the impact of essential factors on the model. The ranges of the factors analysed were calculated by increasing or decreasing them by $20 \%$ (Elbasha \& Messonnier 2004). According to World Health Organization guidelines for cost-effective analysis, the willingness-to-pay (WTP) was set to $\$ 20301.00$ per year, which is $3 \times$ GDP per capita of China (Murray et al. 2000; Eichler et al. 2004). In addition, probabilistic sensitivity analysis was performed by conducting a second-order Monte Carlo simulation to estimate different optimal strategies with varied WTP thresholds (Price \& Briggs 2002).

\section{RESULTS}

\section{Effectiveness}

According to the results of E3805 study, the additional docetaxel has significantly improved the clinical outcome combined with ADT, especially for patients with highvolume disease (Sweeney et al. 2015). Hence, the beneficial outcomes contributed to a gain of 0.48 QALYs for the docetaxel and ADT combination group (2.74 QALYs versus 2.26 QALYs). For the patients with high-volume disease, the effectiveness for docetaxel and ADT combination group was 2.34 QALYs compared to 1.65 QALYs in the ADT alone group, which was 0.69 QALYs longer. The details are listed in Table 2.

\section{Costs}

Treatment associated costs were defined as per month per patient in one transition cycle from the Chinese societal perspective, which was calculated according to the treatment duration and total number of treatment cycles 
collected from the E3805 study, as listed in Table 2. Cost for PFS state was far more than the PD state in both groups. In detail, costs for PFS state, including regimen fees (docetaxel, ADT, dexamethasone), costs of hospitalisation, necessary tests for efficacy/safety evaluation during the treatments, grade 3-4 AEs-related costs, and calcium carbonate and vitamin D costs, were \$24 035.64 in the combined group and $\$ 9916.45$ in the ADT alone group; costs for PD state were $\$ 3051.13$ in the combined group and $\$ 4353.40$ in the $\mathrm{ADT}$ alone group. In total, the incremental cost was $\$ 12816.93$ in the combined group

Table 2. Results of the cost-effectiveness analysis

\begin{tabular}{|c|c|c|}
\hline & $\mathrm{ADT}+\mathrm{D}$ & $\mathrm{ADT}$ \\
\hline \multicolumn{3}{|l|}{ Cost (\$) } \\
\hline \multicolumn{3}{|l|}{ Costs for PFS per month } \\
\hline Docetaxel & 275.67 & - \\
\hline Dexamethasone & 0.098 & - \\
\hline Hospital & 4.54 & 0 \\
\hline ADT & 310.92 & 310.92 \\
\hline Test & 214.73 & 152.39 \\
\hline $\mathrm{AE}$ & 0.40 & \\
\hline Calcium carbonate & 4.18 & 4.18 \\
\hline Vitamin D & 66.63 & 66.63 \\
\hline Total & 877.18 & 534.12 \\
\hline Costs for PD per month & 172.90 & 216.54 \\
\hline Costs for PFS state & 24035.64 & 9916.45 \\
\hline Costs for PD state & 3051.13 & 4353.40 \\
\hline Total costs & 27086.78 & 14269.85 \\
\hline Incremental costs & 12816.93 & \\
\hline $\begin{array}{l}\text { Effectiveness (QALYs) } \\
\text { effectiveness for PFS state }\end{array}$ & 1.85 & 1.26 \\
\hline Effectiveness for PD state & 0.88 & 1.01 \\
\hline Total effectiveness & 2.74 & 2.26 \\
\hline Incremental effectiveness & 0.48 & \\
\hline Cost/effectiveness & 9885.69 & 6314.09 \\
\hline Incremental cost/effectiveness & 26701.94 & \\
\hline
\end{tabular}

$\mathrm{AE}$, adverse event; $\mathrm{PFS}$, progression-free survival; $\mathrm{PD}$, progressive disease; QALY, quality-adjusted life year; ADT, androgendeprivation therapy; $\mathrm{D}$, docetaxel.

Table 3. Adverse events-related costs of metastatic prostate cancer patients treated with docetaxel and ADT combination

\begin{tabular}{lcccc}
\hline Items & $\begin{array}{c}\text { Grade } \\
3(N)\end{array}$ & $\begin{array}{l}\text { Grade } \\
4(N)\end{array}$ & $\begin{array}{l}\text { Cost for } \\
\text { grade 3 }(\$)\end{array}$ & $\begin{array}{c}\text { Cost for } \\
\text { grade 4 }(\$)\end{array}$ \\
\hline Allergic reaction & 7 & 1 & 3.78 & 1.62 \\
Fatigue & 16 & 0 & 0.00 & 0.00 \\
Diarrhoea & 4 & 0 & 13.64 & 0.00 \\
Stomatitis & 2 & 0 & 31.28 & 0.00 \\
Neuropathy, motor & 2 & 0 & 0.00 & 0.00 \\
Neuropathy, sensory & 2 & 0 & 0.00 & 0.00 \\
Thromboembolism & 1 & 2 & 10.54 & 63.25 \\
Sudden death & 0 & 0 & 0.00 & 0.00 \\
Anaemia & 4 & 1 & 56.66 & 42.49 \\
Thrombocytopenia & 0 & 1 & 0.00 & 548.49 \\
Neutropenia & 12 & 35 & 433.35 & 3791.84 \\
Febrile neutropenia & 15 & 9 & 541.69 & 975.04 \\
Infection with & 5 & 4 & 180.56 & 433.35 \\
$\quad$ neutropenia & & & & \\
ADT, androgen-deprivation therapy. &
\end{tabular}

$\mathrm{ADT}$, androgen-deprivation therapy. compared with the ADT alone group $(\$ 27086.78$ versus $\$ 14$ 269.85). Information regarding grade 3-4 AEs analysed in the model are illustrated in Table 3.

\section{Cost-effectiveness analysis}

Evidently, no strategy clearly dominated the others, which means that although docetaxel and ADT combination group was more effective compared to the ADT alone group, the strategy was also more costly. That is, the docetaxel and ADT combination group spent $\$ 9885.69$ per QALY compared with $\$ 6314.09$ per QALY for the ADT alone group. In the base-case analysis, the ICER of docetaxel and ADT combination group compared with the ADT alone group was \$ 26701.94 per QALY, which was far more than the commonly accepted WTP threshold (\$20 301 per QALY in China) (Fig. 2A). Generally, incremental cost-effectiveness scatterplots of ADT alone group were distributed above the WTP (Fig. 3A).

For the subgroup of patients with high-volume disease, the docetaxel and ADT combination group spent $\$ 11077.37$ per QALY compared with $\$ 6844.42$ per QALY for the ADT alone group, and the ICER of the docetaxel and ADT combination group compared with the ADT alone group was \$21 199.63 per QALY, which also exceeded the WTP threshold (Fig. 2B). The incremental cost-effectiveness scatterplots of patients with highvolume disease were almost the same as the overall analysed population (Fig. 3B).

\section{Sensitivity analysis}

A one-way sensitivity analysis was applied, which provides an insight into the responsiveness of the model results to the changes of parameters analysed in the study. The robustness of the results was also tested. The variables in the sensitivity analysis varied at a range of $\pm 20 \%$.

Results of the sensitivity analysis depicted that most factors showed influences on the model results including the costs of docetaxel, ADT, vitamin D and calcium carbonate. Changes in costs for docetaxel had the greatest impact on the results of the analysis. As the value of docetaxel changed from $\$ 220.54$ to $\$ 330.81$, ICER (docetaxel and $\mathrm{ADT}$ combination group versus the $\mathrm{ADT}$ alone group) ranged significantly from $\$ 23830.53$ to $\$ 30206.81$ per QALY, and the cost/effect changed from $\$ 9340.39$ to $\$ 10443.81$ per QALY for the docetaxel plus ADT group. Additionally, the cost-effectiveness analysis was also sensitive to the test costs of the two groups and hospital fees (Fig. 4). 

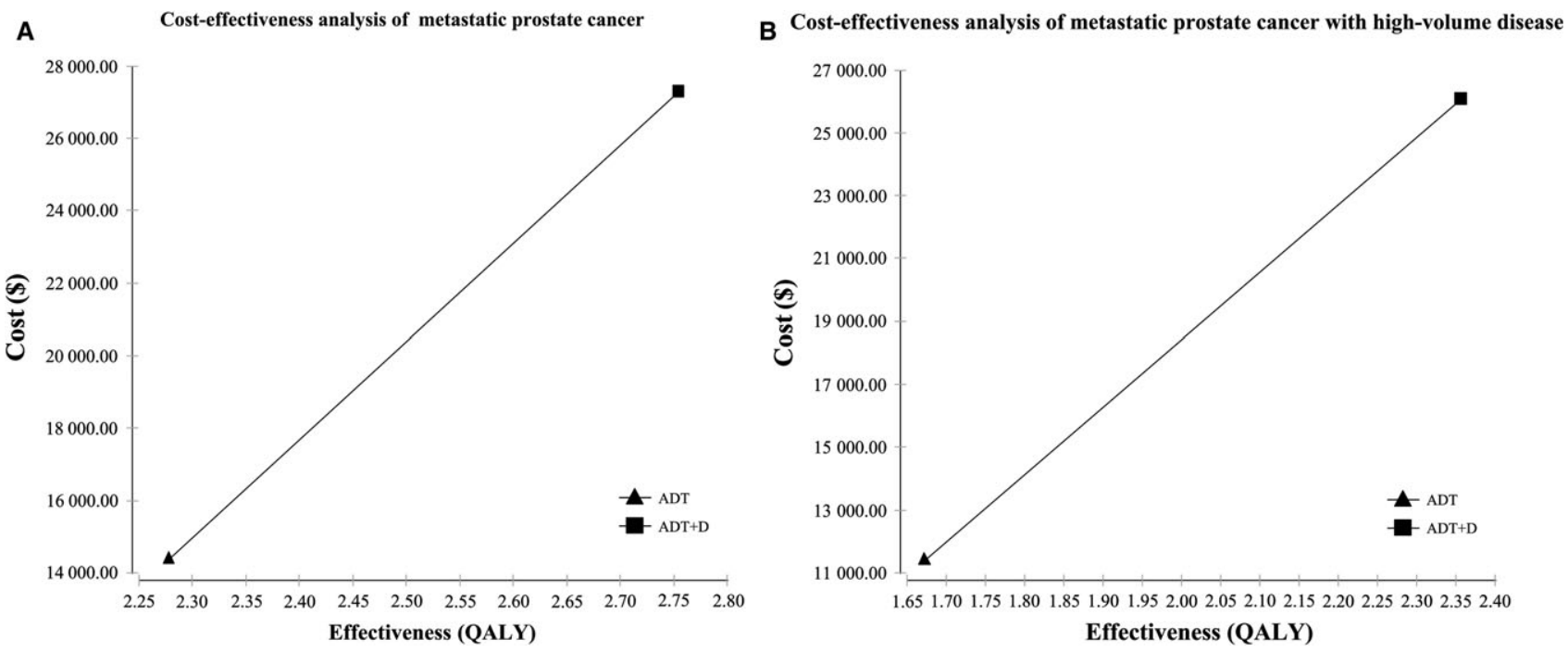

Figure 2. Cost-effectiveness pictured with two groups. Two groups were analysed: group 1, patients with metastatic prostate cancer treated with $\mathrm{ADT}+\mathrm{D}$; group 2, patients with metastatic prostate cancer treated with $\mathrm{ADT}$. ADT, androgen-deprivation therapy; $\mathrm{D}$, docetaxel; QALY, quality-adjusted life years. (A) Cost-effectiveness analysis of whole patients' population. (B) Cost-effectiveness analysis of patients with high-volume disease.
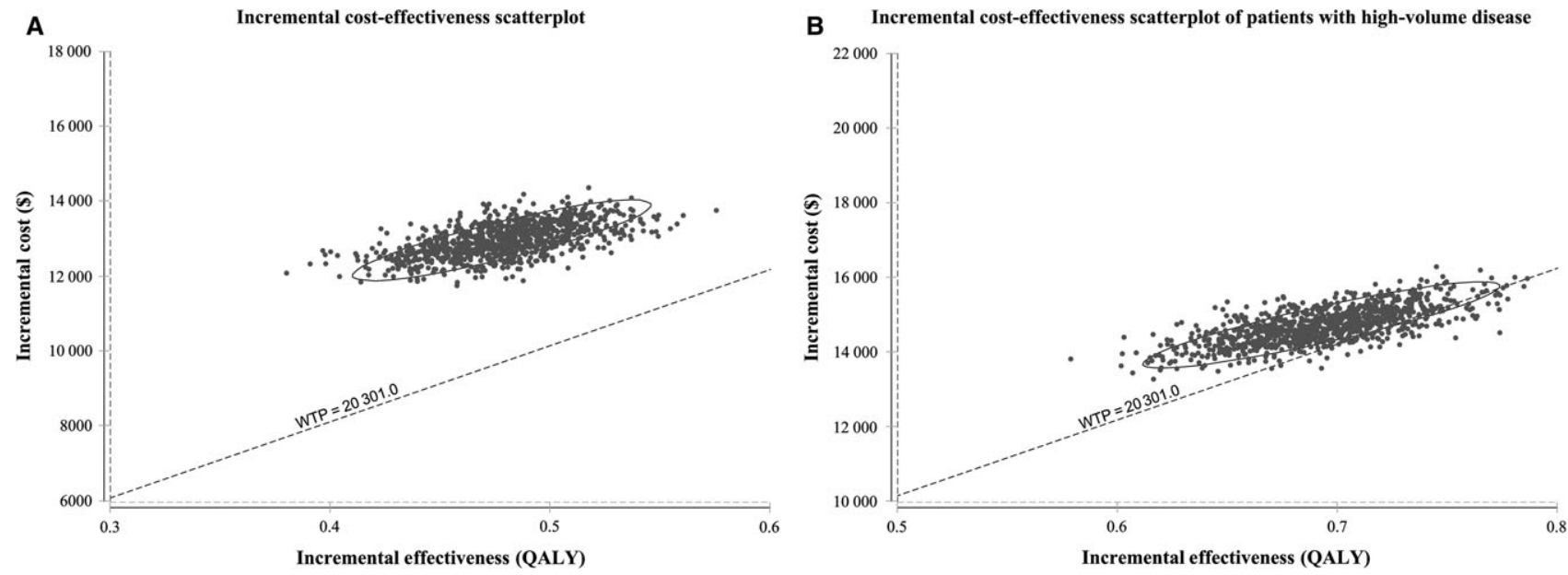

Figure 3. Incremental cost-effectiveness scatterplot of two groups. Two groups were analysed: group 1, patients with metastatic prostate cancer treated with ADT $+\mathrm{D}$; group 2, patients with metastatic prostate cancer treated with ADT. Cost-effectiveness distribution of two groups. ADT, androgen-deprivation therapy; D, docetaxel. (A) Incremental cost-effectiveness scatterplot of whole patients. (B) Incremental cost-effectiveness scatterplot of patients with high-volume disease.

\section{Probabilistic sensitivity analysis (acceptability frontier)}

A probabilistic sensitivity analysis was performed to take into account the uncertainty surrounding estimated parameters. In the cost-effectiveness acceptability curve, the probability of being cost-effective is represented as a function of increasing levels of WTP. With the respect to WTP, as the value varied from $\$ 0$ to $\$ 40602.00$, the acceptable proportion of the ADT alone group was decreased, whereas the docetaxel and ADT combination group was increased (Fig. 5).

\section{DISCUSSION}

The OS of patients with metastatic castration-resistant prostate cancer has achieved much improvement with the development of next-generation cytotoxic chemotherapy, novel hormonal therapies such as enzalutamide and abiraterone acetate, immunotherapy (sipuleucel-T, ipilimumab) and radiopharmaceutical agents radium-223 (Mehta \& Armstrong 2016). However, few studies have investigated how to improve the clinical outcomes in men with metastatic hormone-sensitive disease. The European 
Tornado analysis (ICER)

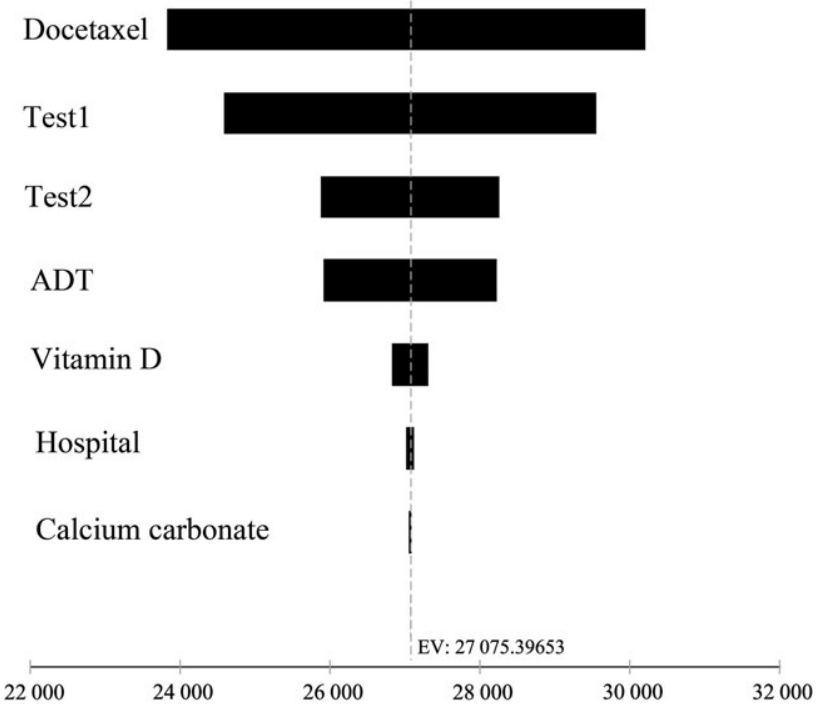

Figure 4. Tornado Diagram of metastatic prostate cancer. Tornado diagram summarised the results of one-way sensitivity analysis to identify model variables associated with the two strategies in the treatment of metastatic prostate cancer. The influential factors were listed descending with the variation of value. ADT, androgen-deprivation therapy; Test1, test costs for ADT plus docetaxel group; Test2, test costs for ADT along group; Hospital, hospital fees.

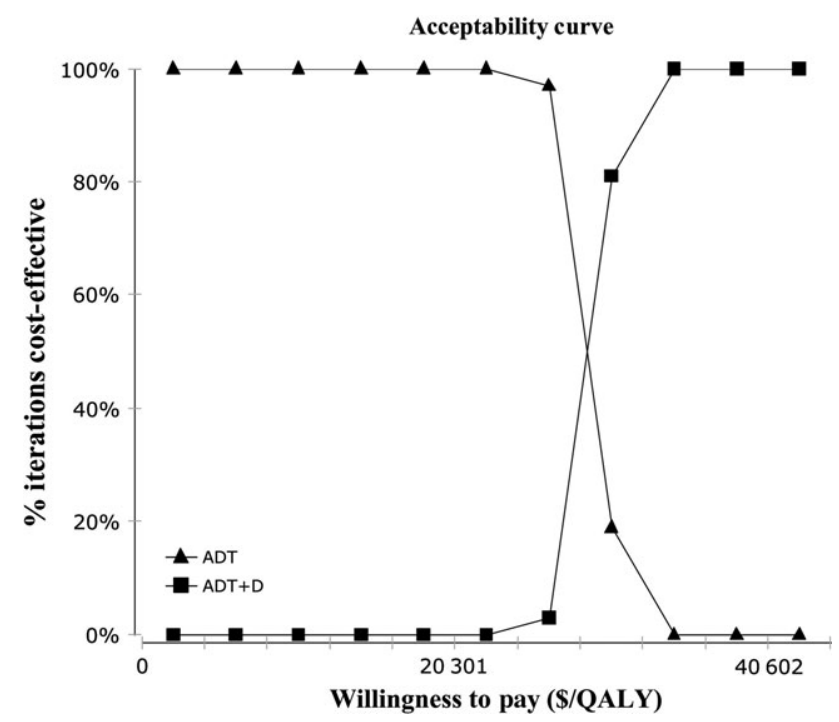

Figure 5. Probabilistic sensitivity analysis (acceptability frontier). The cost-effectiveness acceptability frontier shows the probability of strategies being cost-effective in two strategies. For different willingness-to-pay thresholds, the proportion for $\mathrm{ADT}$ was decreased, while the ADT $+\mathrm{D}$ was increased. ADT, androgen-deprivation therapy; $\mathrm{D}$, docetaxel.

GETUG-AFU-15 study was the first to assess the use of docetaxel in men with metastatic hormone-sensitive prostate cancer (Gwenaelle et al. 2013). Despite the improvement in PFS, the study found no statistically significant difference in the primary end point of OS between the two groups ( $\mathrm{HR}=0.9 ; P=0.44)$. In addition, there was no significant difference in OS for either the high- or lowvolume disease subgroups.

More recently, however, the E3805 study showed a significantly improved OS in metastatic hormone-sensitive prostate cancer patients treated with docetaxel plus ADT compared with ADT alone, especially in those with high-volume disease. These finding led to the update of the National Comprehensive Cancer Network (NCCN) clinical practice guidelines on the treatment of highvolume, ADT-naïve, metastatic hormone-sensitive prostate cancer. With the additional chemotherapy, the medical burden will no doubt be increased dramatically, and it is of great importance to make a preferred treatment recommendation for patients with metastatic hormone-sensitive prostate cancer, especially in a resource-limited country such as China.

In this cost-effectiveness study, we found that chemotherapy with 6 cycles of docetaxel with concurrent ADT is not an affordable choice for patients with metastatic hormone-sensitive prostate cancer at the Chinese societal perspective. Using a decision analytic model, we estimated that the docetaxel and ADT combination group spent $\$ 9885.69$ per QALY compared with $\$ 6314.09$ per QALY for the ADT alone group. Docetaxel improves the survival of the patients by 0.48 QALY at additional cost of $\$ 12$ 816.93. For patients with high-volume disease, the increased cost and effectiveness were \$14 627.75 and 0.69 QALYs in the docetaxel plus ADT group versus the ADT alone group respectively, and the ICER was \$21 199.63 per QALY. These ICERs were higher than the commonly accepted WTP threshold of \$20 301 per QALY in China. Thus, the results indicate that ADT plus docetaxel is not a cost-effective regimen for metastatic hormone-sensitive prostate cancer in China, even for patients with highvolume disease. Of note, docetaxel and growth factor generics have the advantage of lower price, which was the most sensitive factor affecting the results of the current analysis model. However, currently no survival or $\mathrm{AE}$ results have been published of docetaxel generics in patients with metastatic hormone-sensitive prostate cancer. If patients' effects and tolerance to the generics is the same as the originator, the lower price may lead to a more cost-effective treatment strategy. Furthermore, we realised that chemotherapy with docetaxel made the cost much higher than ADT alone, not only because of the expense of the chemotherapy agent itself but also the fees related to hospitalisation, AEs and necessary tests for safety evaluation during the treatments. Notably, there was one treatment-related death among patients receiving 
ADT plus docetaxel, although the E3805 study reported few grade 3-4 haematological AEs (Sweeney et al. 2015). There is a possibility that AEs were underestimated as blood counts were not monitored routinely between chemotherapy cycles (Fizazi et al. 2015). It has been reported that the toxicity of docetaxel is mediated, in part, by hormonal mechanisms (Fitzpatrick \& Ronald 2014). Hence, cost related with AEs might be underestimated.

This cost-effectiveness analysis, to the best of our knowledge, is the first study to investigate whether chemotherapy combined with ADT is a cost-effective option for metastatic hormone-sensitive prostate cancer. Several cost-effective analyses have been done on similar patient populations on various ADT treatments. First, combined androgen blockade therapy with bicalutamide was confirmed to be cost-effective when compared with LH-RH monotherapy in stage D2 prostate cancer (Penson et al. 2005). Then, using modelling with meta-analysis of comparative survival data, another study concluded that leuprorelin $22.5 \mathrm{mg}$ was a more cost-effective treatment than leuprorelin $11.25 \mathrm{mg}$, triptorelin $11.25 \mathrm{mg}$, buserelin $9.9 \mathrm{mg}$ and goserelin $10.8 \mathrm{mg}$ (Iannazzo et al. 2011). Later, researchers from the UK showed that degarelix is unlikely to be cost-effective compared to triptorelin plus shortterm antiandrogen (Lu et al. 2012). All these cost-effectiveness analyses assessed the value of different ADT for patients with metastatic hormone-sensitive prostate cancer, but cannot supply decision-making information on chemotherapy.

However, this cost-effective analysis has several limitations. First, the clinical information was from the American study E3805, while cost data were collected from a single Chinese hospital. Furthermore, we conducted a cost-effectiveness acceptability frontier analysis, which showed the probability of strategies being costeffective. For decision-makers in other countries with different WTP thresholds, different strategies might be optimal. Second, no data of quality of life were available from the E3805 study so utilities for the state of PFS and PD were referenced with previous published research, which may influence the calculation of QALYs. Finally, our analysis did not take into account the costs of AEs caused by the second-line therapy because no information was available.

In summary, our study showed that despite a longer survival time, 6 cycles of docetaxel combined with $\mathrm{ADT}$ is not a preferred cost-effective treatment for metastatic hormone-sensitive prostate cancer in the Chinese setting, even for patients with high-volume disease. A reduction in the price of docetaxel and further research are needed to improve the cost-effectiveness for metastatic hormonesensitive prostate cancer; further studies might include distinguishing patients with de novo metastases at diagnosis from those who developed metastases following treatment of localised disease, considering combination of ADT with novel treatment strategies, and investigating concurrent or sequential ADT and chemotherapy. Nevertheless, this analysis provides evidence from an economic perspective regarding the addition of chemotherapy to hormone therapy in the treatment of prostate cancer. The conclusions will be helpful for the decision-making of physicians, patients and healthcare management structures.

\section{ACKNOWLEDGEMENTS}

The authors thank Dr. Kirsten Herold from the School of Public Health, University of Michigan for her helpful revisions of the manuscript. The authors also acknowledge Dr. Jeffrey Montgomery in Department of Urology, University of Michigan for his fruitful discussions for the study.

\section{FUNDING}

The authors declare no funding.

\section{DISCLOSURE}

The authors declare no conflict of interest.

\section{REFERENCES}

Center M.M., Jemal A., Lortet-Tieulent J., Ward E., Ferlay J., Brawley O. \& Bray F. (2012) International variation in prostate cancer incidence and mortality rates. European Urology 61, 1079-1092.

Eichler H.G., Kong S.X., Gerth W.C., Mavros P. \& Jonsson B. (2004) Use of cost-effectiveness analysis in health-care resource allocation decision-making: how are cost-effectiveness thresholds expected to emerge? Value Health 7, 518-528.

Elbasha E.H. \& Messonnier M.L. (2004) Cost-effectiveness analysis and health care resource allocation: decision rules under variable returns to scale. Health Economics 13, 21-35.

Fitzpatrick J.M. \& Ronald D.W. (2014) Taxane mechanisms of action: potential implications for treatment sequencing in metastatic castration-resistant prostate cancer. European Urology 65, 1198-1204.
Fizazi K., Jenkins C. \& Tannock I.F. (2015) Should docetaxel be standard of care for patients with metastatic hormonesensitive prostate cancer? Pro and Contra. Annals of Oncology 26, 1660-1667.

Gwenaelle G., Karim F., Florence J., Stéphane O., Franck P., Benjamin E., Igor L., Remy D., Ivan K. \& Brigitte L. (2013) Androgen-deprivation therapy alone or with docetaxel in non-castrate metastatic prostate cancer (GETUGAFU 15): a randomised, open-label, 
phase 3 trial. The Lancet Oncology 14, 149-158.

Heijnsdijk E.A., De Carvalho T.M., Auvinen A., Zappa M., Nelen V., Kwiatkowski M., Villers A., Paez A., Moss S.M., Tammela T.L., Recker F., Denis L., Carlsson S.V., Wever E.M., Bangma C.H., Schroder F.H., Roobol M.J., Hugosson J. \& De Koning H.J. (2015) Cost-effectiveness of prostate cancer screening: a simulation study based on ERSPC data. Journal of the National Cancer Institute 107, 366.

Hongmei Z., Rongshou Z., Yuming G., Siwei Z., Xiaonong Z., Ning W., Limei Z., Jingao T., Jianguo C. \& Kuangrong W. (2015) Cancer survival in China, 2003-2005: a population-based study. International Journal of Cancer 136, 1921-1930.

Iannazzo S., Pradelli L., Carsi M. \& Perachino M. (2011) Cost-effectiveness analysis of LHRH agonists in the treatment of metastatic prostate cancer in Italy. Value Health 14, 80-89.

Jr G.W., Coffey D., Karr J.P., Chiarodo A., Epstein J., Mcneal J.E. \& Miller G. (1987) Diagnostic nomenclature for prostate cancer. Journal of Urology 138, 1436.

Lu L., Peters J., Roome C. \& Stein K. (2012) Cost-effectiveness analysis of degarelix for advanced hormonedependent prostate cancer. BJU International 109, 1183-1192.

Maluf F.C., Smaletz O. \& Herchenhorn D. (2012) Castration-resistant prostate cancer: systemic therapy in 2012. Clinics 67, 389-394.

Mehta A.R \& Armstrong A.J. (2016) Tasquinimod in the treatment of castrate-resistant prostate cancer-current status and future prospects. Therapeutic Advances in Urology 8, 9-18.

Murray C.J., Evans D.B., Acharya A. \& Baltussen R.M. (2000) Development of WHO guidelines on generalized costeffectiveness analysis. Health Economics 9, 235-251.

National Comprehensive Cancer Network (NCCN) (2015) Clinical Practice Guidelines in Oncology-Prostate cancerv.1.2015. Available at: http://www. nccn.org/professionals/physician_gls/pdf/ prostate.pdf (accessed September 2015).

Nishiyama T. (2008) Intra-prostatic androgen levels during various androgenblockade regimens. Best Practice and Research. Clinical Endocrinology and Metabolism 22, 229-241.

Penson D.F., Ramsey S., Veenstra D., Clarke L., Gandhi S. \& Hirsch M. (2005) The cost-effectiveness of combined androgen blockade with bicalutamide and luteinizing hormone releasing hormone agonist in men with metastatic prostate cancer. Journal of Urology 174, 547-552.

Petrou P.K. \& Talias M.A. (2014) Costeffectiveness of sorafenib compared to best supportive care in second line renal cell cancer from a payer perspective in Cyprus. Expert Review of Pharmacoeconomics and Outcomes Research 14, 131-138.

Potosky A.L., Miller B.A., Albertsen P.C. \& Kramer B.S. (1995) The role of increasing detection in the rising incidence of prostate cancer. JAMA 273, 548-552.

Price M.J. \& Briggs A.H. (2002) Development of an economic model to assess the cost effectiveness of asthma management strategies. Pharmacoeconomics 20, 183-194.

Purmonen T., Martikainen J.A., Soini E.J., Kataja V., Vuorinen R.L. \& KellokumpuLehtinen P.L. (2008) Economic evaluation of sunitinib malate in secondline treatment of metastatic renal cell carcinoma in Finland. Clinical Therapeutics 30, 382-392.

Siegel R.L., Fedewa S.A., Miller K.D., Goding-Sauer A., Pinheiro P.S., Martinez-Tyson D. \& Jemal A. (2015) Cancer statistics for Hispanics/Latinos. CA: A Cancer Journal for Clinicians 65, 5-29.

Sweeney C.J., Chen Y.H., Carducci M., Liu G., Jarrard D.F., Eisenberger M., Wong Y.N., Hahn N., Kohli M., Cooney M.M., Dreicer R., Vogelzang N.J., Picus J., Shevrin D., Hussain M., Garcia J.A. \& Dipaola R.S. (2015) Chemohormonal therapy in metastatic hormone-sensitive prostate cancer. New England Journal of Medicine 373, 737-746.

Tannock I.F., De Wit R., Berry W.R., Horti J., Pluzanska A., Chi K.N., Oudard S., Theodore C., James N.D., Turesson I., Rosenthal M.A. \& Eisenberger M.A. (2004) Docetaxel plus prednisone or mitoxantrone plus prednisone for advanced prostate cancer. New England Journal of Medicine 351, 1502-1512.

Wu J.N., Fish K.M., Evans C.P., Devere White R.W. \& Dall'era M.A. (2014) No improvement noted in overall or causespecific survival for men presenting with metastatic prostate cancer over a 20-year period. Cancer 120, 818-823. 